\title{
Los residentes en las unidades de cuidados intensivos, ¿cuál es su percepción de la formación que reciben?
}

\author{
Guillermo Vázquez-Mata, Manuel Rodríguez-Elvira, Luis Rucabado-Aguilar, Ángel García-Alcántara, \\ Francisco Murillo-Cabezas, Pedro Navarrete-Navarro, Ana Guillamet-Lloveras, Juan Roca-Guiseris
}

Objetivo. Este trabajo intenta responder a la pregunta de cuál es la visión que tienen los residentes de su formación en las unidades de cuidados intensivos (UCI).

Sujetos y métodos. Hemos realizado un estudio cualitativo tipo grounded theory. Los participantes son residentes de cualquier especialidad que estuviesen trabajando en las UCI durante el estudio. El diseño tiene tres partes: percepción subjetiva de los residentes de aquellos aspectos que ellos consideran más útiles para su formación, priorización de las actividades regulares más características de las UCI y entrevistas semiestructuradas con informadores claves.

Resultados. Nuestro trabajo identifica que los residentes consideran como eje de su formación la práctica clínica a 'pie de cama' desarrollada con autonomía y apoyada en una buena tutorización. Paralelamente, otras competencias nucleares como la investigación, la comunicación en situaciones complejas, el trabajo en equipo o la gestión de recursos están infravaloradas, mientras que otras como la seguridad del paciente o la bioética no se han detectado en las respuestas de los residentes.

Conclusión. La percepción de los residentes sobre formación durante su estancia en las UCI adolece de algunas carencias, dado que ciertos aspectos claves de la medicina actual no se percibien como prioridades en dicha formación.

Palabras clave. Competencias. Entrenamiento. Investigación cualitativa. Residentes. UCI.

\section{Residents in an Intensive Care Unit, what perception they have on their training?}

Aim. Our work tries to answer the following question: what is the perception of residents on their training in the Intensive Care Units (ICU)?

Subjects and methods. We have conducted a qualitative study based on grounded theory. Participants are residents from different specialties working in the ICU of four hospitals of our National Health Service. The study consist of three parts: resident's subjective perception of those aspects most appreciate in their clinical practice; resident's prioritizations of routine ICU's activities, and semi-structured interviews with key informants.

Results. According to the resident's opinions, the clinical practice at the beside of patients, and carried out with autonomy and with a good tutoring support are central to their training; nevertheless some central competencies such as research, difficult communication, team work or resource management are undervalued, while others such as patient safety or bioethics are absent from their comments.

Conclusions. Our work highlight that resident's perception about their training during they compulsory period in ICU has some shortcoming, because some key aspects of current medicine are not perceived as priorities in their training.

Key words. Competencies. ICU. Qualitative research. Residents. Training.

\section{Introducción}

La formación de los médicos residentes en las Unidades de Cuidados Intensivos (UCI) persigue prepararlos para una buena práctica clínica basada en el trabajo en equipo multidisciplinar; así mismo promueve la investigación, enseña a trabajar dentro de las dimensiones del profesionalismo, entrena en una buena comunicación en cualquier circunstancia y enseña a gestionar con calidad los recursos que dependan de ellos [1,2]. Paralelamente, la necesidad del reciclaje permanente para adaptarse a escenarios cambiantes hace que el autoaprendizaje y el pensamiento crítico sean imprescindibles en su
Fundación Educación Médica (G. Vázquez-Mata). Hospital Universitario Virgen de las Nieves; Granada (M. Rodríguez-Elvira, P. Navarrete-Navarro, J. RocaGuiseris). Hospital General de Jaén (L. Rucabado-Aguilar). Hospita Clínico Virgen de la Victoria; Málaga (A. García-Alcántara). Hospital Universitario Virgen del Rocío; Sevilla (F. Murillo-Cabezas) Escuela de Enfermería Virgen de las Nieves; Granada, España (A. Guillamet-Lloveras).

Correspondencia:

Dr. Guillermo Vázquez Mata.

Fundación Educación Médica. Amapola, 6. E-18151 Ojígares (Granada).

E-mail:

vazquez95@gmail.com

(C) 2011 Educación Médica 
formación [3,4]. Este conjunto de características no sólo son válidas para los residentes que han elegido medicina intensiva, sino también para aquellos que en sus especialidades contemplan rotaciones por las UCI [1]. Para dar respuesta a estas necesidades, la Sociedad Española de Medicina Intensiva, Críticos y Unidades Coronarias [1] ha publicado un conjunto de competencias, tanto específicas como transversales, que deberían dominarse para trabajar con pacientes críticos. Este escenario puede considerarse ambicioso y complejo,

Las publicaciones sobre la formación de los residentes en las UCI, además de ser relativamente escasas, están planteadas desde la perspectiva de las plantillas de las UCI o de sus directores y se han desarrollado con metodologías cuantitativas que centran los resultados en aspectos concretos; sin embargo, no hemos encontrados estudios en que el propio residente exprese su visión personal de su formación en la UCI [5]. Nuestro estudio ha intentado aportar esta última perspectiva, es decir, ¿cuál es la visión que tienen los residentes de su formación en la UCI? Para contestar a esta pregunta nos hemos fijado tres objetivos: identificar aquellos aspectos que los residentes perciben como mas sobresalientes en su formación, saber cómo valoran las actividades rutinarias que se desarrollan en una UCI desde dicha perspectiva y conocer las características del médico que mejor les forma.

\section{Sujetos y métodos}

Para responder a la pregunta planteada, hemos seleccionado una metodología cualitativa tipo grounded theory [6]; el método ha sido concurrente, es decir, las facetas del estudio se aplicaron simultáneamente y a la vez se ha generado su visión desde varios ángulos para poder triangular las observaciones realizadas.

El estudio es de base hospitalaria, habiéndose realizado en cuatro servicios de medicina intensiva de Andalucía: el Hospital Universitario Virgen de las Nieves (Granada), el Hospital Clínico Virgen de la Victoria (Málaga), el Complejo Hospitalario de Jaén y el Hospital Universitario Virgen del Rocío (Sevilla), todos ellos pertenecientes al Sistema $\mathrm{Na-}$ cional de Salud. Dos expertos en medicina intensiva y un experto en investigación cualitativa han trabajado con metodología de consenso, tanto para realizar propuestas como para analizar los datos aportados en el estudio. Los participantes eran residentes de cualquier especialidad y año de residen- cia que estuvieran trabajando en la UCI en el momento de realizarse el estudio; su participación fue voluntaria y anónima. Las definiciones utilizadas son:

- Categoría: concepto identificado en las respuestas de los residentes.

- Actividad: acción tendente a conseguir un fin.

- Competencia: habilidad necesaria para realizar una actividad profesional básica

- Competencia nuclear: corresponde a una competencia genérica que engloba todas las competencias específicas y transversales relacionadas con ella $[1,7]$.

- Dimensión: función médica que requiere conjuntar competencias de diferentes competencias nucleares.

El estudio consta de tres partes:

- La primera parte comprende la percepción subjetiva de los residentes de aquellas actividades que consideraban más útiles para su formación. El grupo de expertos que puso en marcha el trabajo propuso dos preguntas abiertas: 'en tu actividad en la UCI, ¿qué consideras más útil para tu formación?' y 'en tu relación con médicos de plantilla, ¿cuál es el perfil del médico que enseña mejor?'. Las preguntas abiertas se pasaron en formato escritura a los residentes en una sesión presencial. En las respuestas relacionadas con la primera pregunta, los expertos identificaron las categorías que contenían, que a su vez se agruparon en actividades relacionadas con un mismo fin. Respecto a la segunda pregunta, los expertos identificaron las categorías, las agruparon en competencias y éstas, a su vez, se reagruparon en tres dimensiones.

- La segunda parte fue la priorización de las actividades más características de las UCI. Los expertos consensuaron una lista con las 10 actividades que consideraron que definían mejor las actividades básicas y rutinarias de la UCI. Esta lista se pasó a los residentes en la misma sesión presencial que las preguntas abiertas y se les pidió que las priorizaran con una escala de 10 puntos, asignando 10 puntos a la que consideraban más útil para su formación, y 1, punto a la menos útil; se pidió que no se repitieran puntuaciones. La suma de las puntuaciones que cada actividad recibió permitió escalonarlas de más a menos útil.

- Finalmente se realizaron varias entrevistas semiestructuradas con informadores claves para mejorar la compresión de las respuestas de los residentes. 
La triangulación consistió en comprobar por parte de los expertos las coincidencias entre las respuestas que se obtuvieron tanto con las preguntas abiertas como en la priorización de actividades de la UCI.

\section{Resultados}

Participaron 49 residentes de los 62 que trabajaban en ese momento en las UCI; de éstos, 34 (69,3\%) eran residentes de medicina intensiva, y el resto, 15 (30,6\%), de otras especialidades como anestesia o medicina interna.

En relación a las respuestas de la pregunta libre 'qué consideras más útil para tu formación', se identificaron 131 categorías que se agruparon en 10 actividades (Tabla I); entre las categorías identificadas no figuran conceptos directamente relacionados con la comunicación en cualquiera de sus aspectos, la seguridad del paciente y prevención de errores, la calidad clínica o la bioética.

Los residentes priorizaron las actividades que el grupo de expertos propuso como características de las UCI, como se recoge en la tabla II. La diferencia de puntuación entre las actividades clínicas y las relacionadas con la investigación llegaba a 170 puntos.

Los residentes consideraron que, para su formación, las características más útiles de los médicos con los que trabajaban a pie de cama eran las que se reflejan en la tabla III.

Los informadores claves coinciden en señalar:

- La palabra 'tutorización' no se refiere a la figura oficial del tutor que gestiona la actividad de los residentes en las UCI, sino a la figura del médico que lleva las camas en las que ellos trabajan.

- La retroalimentación ofeedback se considera como una evaluación de las actividades que realizan.

- Las sesiones clínicas y los cambios de guardia o turnos los describen como enumeración de datos, con poca interactividad en relación a los residentes.

- La investigación en sí y otras actividades relacionadas con ella no se perciben como prioridades en la formación de los residentes.

- La seguridad del paciente no es motivo de análisis en las actividades a 'pie de cama'.

\section{Discusión}

Nuestro trabajo identifica que los residentes tienen la percepción de que el eje de su formación es la práctica clínica a 'pie de cama', desarrollada con autonomía y apoyada en una buena tutorización por
Tabla I. Actividades formativas propuestas por los residentes.

\begin{tabular}{|c|c|}
\hline Actividades ${ }^{a}$ & $n(\%)$ \\
\hline Llevar enfermos ${ }^{b}$ & $27(55,1)$ \\
\hline Estar tutorizados & $26(53,0)$ \\
\hline Entrenar habilidades ${ }^{c}$ & $21(42,8)$ \\
\hline Tener autonomía & $15(30,6)$ \\
\hline Mejorar la formación d & $15(30,6)$ \\
\hline Participar en actividades ${ }^{\mathrm{e}}$ & $13(26,5)$ \\
\hline Razonar clínicamente ${ }^{f}$ & $8(16,3)$ \\
\hline Investigar & $4(8,1)$ \\
\hline Manejar recursos 9 & $1(2,0)$ \\
\hline Relacionarse con las familias ${ }^{h}$ & $1(2,0)$ \\
\hline \multicolumn{2}{|c|}{$\begin{array}{l}\text { a } \text { Las categorías identificadas en las respuestas se han agrupado en die } \\
\text { actividades; }{ }^{b} \text { Llevar pacientes encamados y el trabajo de las guardias; }{ }^{\mathrm{C}} \mathrm{Ha} \\
\text { bilidades psicomotoras; }{ }^{\mathrm{d}} \text { Cursos normales; }{ }^{\mathrm{e}} \text { Se refieren a sesiones clínicas } \\
\text { bibliográficas; }{ }^{\mathrm{f}} \text { Saber diagnosticar y seleccionar tratamientos; }{ }^{\mathrm{g}} \text { Criterios d } \\
\text { admisión y alta; } \text { h Empatía. }\end{array}$} \\
\hline
\end{tabular}

Tabla II. Priorización de las actividades rutinarias de las unidades de cuidados intensivos.

\begin{tabular}{lc}
\hline Actividades $^{\text {a }}$ & Puntos $^{\text {b }}$ \\
\hline Llevar enfermos & 357 \\
\hline Trabajar en las guardias & 342 \\
\hline Interactuar con el staff & 288 \\
\hline Buscar información & 251 \\
\hline Feedback & 233 \\
\hline Sesión clínica diaria & 227 \\
\hline Información de la guardia & 224 \\
\hline Preparar trabajos & 201 \\
\hline Participar en proyectos de investigación & 187 \\
\hline Asistir a congresos & 179 \\
\hline
\end{tabular}

a Las actividades propuestas por los expertos fueron priorizadas por los residentes según el impacto en su formación. Cada actividad se puntuó entre 10 y 1, no pudiéndose repetir la misma cifra. A mayor puntuación, mayor impacto; b Corresponde a la suma de los puntos asignados a cada actividad por los residentes. 
Tabla III. Características que los residentes valoran en los médicos con los que se relacionan $(n=121)$.

\begin{tabular}{|c|c|c|}
\hline & & $n(\%)$ \\
\hline \multirow{3}{*}{$\begin{array}{l}\text { Como } \\
\text { clínico }^{a}\end{array}$} & Buen clínico ${ }^{b}$ & $13(10,7)$ \\
\hline & Gestiona su tiempo & $5(4,1)$ \\
\hline & Investiga & $2(1,6)$ \\
\hline \multirow{5}{*}{$\begin{array}{l}\text { Como } \\
\text { tutor }{ }^{\mathrm{a}}\end{array}$} & Crea un clima confortable ${ }^{c}$ & $23(19,0)$ \\
\hline & Realiza feedback & $23(19,0)$ \\
\hline & Promueve tu autonomía & $13(10,7)$ \\
\hline & Le gusta enseñar & $12(9,9)$ \\
\hline & Promueve el pensamiento clínico ${ }^{d}$ & $11(9,0)$ \\
\hline \multirow{4}{*}{$\begin{array}{l}\text { Como } \\
\text { profesional }^{a}\end{array}$} & Plantilla júnior e & $6(4,9)$ \\
\hline & Centrado en el paciente & $5(4,1)$ \\
\hline & Trabaja en equipo & $5(4,1)$ \\
\hline & Responsable & $3(2,4)$ \\
\hline
\end{tabular}

a Las respuestas se han agrupado en tres dimensiones que a su vez agrupan las características que los residentes resaltan de los médicos con los que trabajan a 'pie de cama'; ' b Lleva enfermos, hace guardias, está actualizado, razona sus decisiones; ' Escucha activa, reconoce tu trabajo, trato agradable, te anima, te orienta, no examina; ${ }^{\mathrm{d}}$ Diagnóstico, pensamiento crítico/reflexivo; e Residentes de últimos años, especialistas jóvenes.

el médico clínico con el que trabajan; también resaltan que los mejores resultados los tienen cuando dicha tutorización se realiza en un clima de relaciones humanas confortable. Paralelamente, la investigación, el trabajo en equipo, la gestión de recursos o la relación con las familias se valoran por debajo de la práctica clínica, mientras que otras dimensiones, como la seguridad del paciente o la bioética, no se han detectado en las respuestas espontáneas de los residentes. La pregunta que nos planteábamos como punto de partida del trabajo (cuál es la visión que tienen los residentes de su formación en la UCI) muestra, desde la perspectiva de este trabajo, oportunidades de mejora [5].

Llevar enfermos y tener autonomía suponen una serie de competencias, agrupadas en la competencia nuclear 'práctica clínica' [7]. Esto presupone realizar historias clínicas y exploraciones correctas, interpretar técnicas de imagen y de laboratorio, y finalmente, conjuntarlo e interpretarlo; para alcanzar esta última meta se requiere un pensamiento clínico sobre el que basar un plan diagnóstico y un plan terapéutico $[1,4]$. En las UCI hay que añadir el manejo adecuado de un conjunto de equipos tecnológicos que rodean al paciente [1]. La complejidad de esta actividad hace que la tutorización a 'pie de cama' confiera seguridad y autoconfianza $[8,9]$.

Sin embargo, llevar enfermos requiere actualmente otras dimensiones o competencias nucleares [1-3,7] que no hemos identificado entre las categorías que los residentes mencionan en sus respuestas libres. En primer lugar, el trabajo en equipo [10], que representa no sólo la relación diaria con otros profesionales, sino también liderazgo, coordinación y trabajo colaborativo. En segundo lugar, la seguridad de los pacientes [11], dimensión siempre presente en las UCI por el riesgo de error existente [12]. Otras categorías no citadas de manera específica son las relacionadas con la comunicación $[1,3,5]$. Resulta obvio que llevar enfermos supone la realización de la historia clínica, la comunicación con la familia y la realizaron de sus registros; este enfoque, aunque sea correcto, margina muchos aspectos de la comunicación. Así, basta recordar la comunicación en situaciones difíciles como son las malas noticias o las situaciones de conflicto, la exposición en público y la defensa de lo expuesto. Otros aspectos de la comunicación son el consentimiento informado, la participación del enfermo o su familia en la toma de decisiones $[1,3]$ y la comunicación cuando se asume el rol de liderazgo en situaciones criticas [12].

La bioética y la toma de decisiones basadas en ella también están ausentes en los comentarios de los residentes $[1,3,4]$. Todos los residentes deberán tomar en algún momento decisiones relacionadas con la limitación del esfuerzo terapéutico [13], la donación de órganos para trasplantes o el respeto a la autonomía del paciente en su rechazo a determinadas medidas terapéuticas, o incluso decisiones personales relacionadas con conflictos de intereses $[3,14]$. Esta dimensión es un compromiso básico del profesionalismo actual [3].

La investigación [15] se valora relegada a un segundo plano; dado que detrás de la investigación existe un pensamiento analítico y crítico, su baja valoración puede suponer no sólo una carencia en su formación en metodología científica, sino también una carencia para analizar la realidad de su entorno, transferir el conocimiento útil a la cabecera de sus pacientes $[1,2,4]$ y tomar decisiones en condiciones de incertidumbre.

Otro aspecto llamativo de nuestros resultados es una apreciación intermedia o baja de las reuniones clínicas que se hacen de manera regular en las UCI [16]; este problema se cita recurrentemente en la bibliografía internacional y se atribuye a su baja interactividad [16-18]. 
La visión que tienen los residentes del médico que los forma mejor coincide con las características que la bibliografía internacional atribuye al buen tutor [8]; para los residentes, su máximo valor es la habilidad de crear un clima confortable, seguido de una buena práctica clínica. En su perfil, la investigación tampoco está bien valorada. La evaluación de los progresos realizados no se identifica como un valor del tutor, si bien, tal como interpretan los expertos, los residentes consideran la retroalimentación del tutor como una evaluación continua.

La práctica clínica, la autonomía y la tutorización a 'pie de cama' constituyen las bases de la formación especializada. Sin embargo, la no identificación en los comentarios de los residentes de ciertas categorías que puedan identificarse con otras actividades, dimensiones y competencias nucleares puede representar un déficit importante para ellos. Diversas causas pueden explicarlo, pero en último término pueden reflejar la ausencia de este tipo de aproximación a los problemas clínicos diarios en las actividades de la UCI y, por tanto, constituir aspectos mejorables de estos servicios. A esto se puede sumar, por parte de los residentes, la disminución de horas en la cabecera del paciente [19] y un primer año sin responsabilidad objetiva [4], y por parte de las plantillas, una presión asistencial y administrativa que absorbe casi todo su tiempo. También cabe recalcar que muchos de los cursos que reciben sobre las dimensiones y actividades menos valoradas se basan en clases magistrales y, por tanto, con una baja capacidad de retenerse e influir en sus conductas y valoraciones.

En nuestro estudio hemos recurrido a la metodología cualitativa al considerarla idónea para la pregunta que planteábamos. Hay algunas de sus características que conviene destacar. Nuestros datos sólo son válidos en el ambiente en que se han estudiado, es decir, no se pueden generalizar; sin embargo, por las características comunes de las UCI en todo nuestro Sistema Nacional de Salud, es posible que estos resultados puedan encontrarse en otros servicios. Además, los residentes que han participado en el trabajo son de varias especialidades y, por tanto, no se pueden atribuir los resultados como específicamente de medicina intensiva, pero como el objetivo del estudio no eran competencias propias de intensivos sino responder a cómo perciben los residentes su formación en una UCI, los resultados reflejan esta aproximación.

La investigación cualitativa [6], actualmente con una sólida base doctrinal, es poco conocida por los intensivistas. Los interrogantes que este trabajo pone sobre el tapete, como es la ausencia de una presen- cia sólida de muchas de las actividades y dimensiones básicas en la percepción de los residentes, constituyen un reto. Nuestro estudio es un primer bucle de análisis, pero la grounded theory requiere nuevos bucles de análisis que busquen entender el porqué de esta percepción, sus causas, e identificar las mejores estrategias para corregirlas.

En resumen, la metodología cualitativa ha permitido identificar aquellos aspectos que los residentes perciben como más o menos prioritarios y útiles en su formación, y a la vista de las respuestas obtenidas cabe preguntarse si no existe una disociación entre lo que creemos que enseñamos y transmitimos a los residentes y lo que finalmente ellos valoran y transfieren a su práctica clínica diaria.

\section{Bibliografía}

1. Roca J, Pérez JM, Colmenero M, Muñoz H, Alarcón L, Vázquez G. Competencias profesionales para la atención al paciente crítico. Más allá de las especialidades. Med Intensiva 2007; 31: 473-84.

2. Dorman T, Angood PB, Angus DC, Clemmer T, Cohen H, Durbin CG, et al. Guidelines for critical care medicine training and continuing medical education. Crit Care Med 2004; 32: 263-72.

3. Blank L, Kimball H, McDonald W, Merino J. Medical professionalism in the new millennium: a physician chart 15 months later. Ann Intern Med 2003; 183: 839-41.

4. Vázquez G, Murillo-Cabezas F, Gómez J, Martín C, Chaves J, Peinado JL. El examen MIR, su cambio como una opción estratégica. Educ Med 2008; 11: 203-6.

5. Chudgar SM, Cox CE, Que LG, Andolsek K, Knudsen N Clay AS. Current teaching and evaluation methods in critical care medicine: has the Accreditation Council for Graduate Medical Education affected how we practice and teach in the intensive care unit? Crit Care Med 2009; 37: 49-60.

6. Lingard L Albert, M, Levinson W. Qualitative research: grounded theory, mixed methods, and action research. BMJ 2008; 337: 450-61.

7. Palsson R, Kellett J, Lindgren S, Merino J, Semple C, Sereni D. Core competencies of the European internist, a discussion paper. Eur J Intern Med 2007; 18: 104-8.

8. Ramani S, Leincester S. Teaching in the clinical environment. Med Teach 2008; 30: 347-64.

9. Young L, Orlandi A, Galichet B, Heussler H. Effective teaching and learning on the wards: easier said than done? Med Educ 2009; 43: 808-17.

10. Reader T, Fin R, Lauche K, Cuthbertson BH. Non technical skills in the intensive care unit. BJA 2006; 95: 551-9.

11. Weingart S, Wilson R, Gibberd RW, Harrison B. Epidemiology of medical error. BMJ 2000; 320: 774-7.

12. Lighthall G, Barr J, Howard SK, Gellar E, Sowb Y Bertacini, E, et al. Use of a fully simulated intensive care unit environment for critical event management training for internal medicine residents. Crit Care Med 2003; 31: 2434-43.

13. Kuper A, Edden ZN, Etchells E, Shadowitz S, Reeves S. Teaching and learning in morbidity and mortality rounds: an ethnographic study. Med Educ 2010: 44: 559-69.

14. Alfandre D, Rhodes R. Improving ethics education during residency training. Med Teach 2009; 31: 513-7.

15. Maudsley G, Strivens J. Science, critical thinking and competence for Tomorrow's Doctors. A review of terms and concepts. Med Educ 2000; 34: 53-60. 
16. Swanwick T, Morris C. Shifting conceptions of learning in the workplace. Med Educ 2010; 44: 538-9.

17. Cavis D, Thomson MA, Freemantle N, Wolf F, Mazmanian P, Taylor A. Do conferences, workshops, round and other traditional continuing education activities change physician behaviour or health care outcomes? JAMA 1999; 282: 867-74.
18. Walton JM, Steinert Y. Patterns of interaction during rounds: implications for work-based learning. Med Educ 2010: 44: 550-8.

19. Fletcher KE, Davis SQ, Underwood W, Mangrulkar RS, McMahon LF, Saint S. Systematic review: effects of resident work hours on patient safety. Ann Intern Med 2004; 141: 851-7. 